\title{
L'adiaspiromycose
}

\section{chez les petits mammifères sauvages en Bulgarie}

\author{
par T. GUEGNOV *, ZI. ZLATANOV ** et Jan PROKOPIC *** \\ * Académie agronomique «G. Dimitrov», Chaire de zoologie, Sofia, Bulgarie, \\ ** Station Républicaine Antiépidémique, 3, rue Vérila, Sofia, Bulgarie, \\ et *** Parasitologicky ustav C̆SAV, Praha 6-Flemingovo, nam 2, CSSR
}

\section{Résumé}

A la suite des recherches exécutées sur 2.057 exemplaires de mammifères sauvages provenant de 3 régions de Bulgarie et appartenant à 28 espèces, nous avons trouvé des adiaspores d'Emmonsia crescens chez 10 espèces: Crocidura leucodon, Crocidura suaveoles, Neomys anomalus, Sorex minutus, Apodemus agrarius, Apodemus flavicollis, Apodemus sylvaticus, Mus musculus, Microtus arvalis, Clethrionomys glareolus. Parmi elles, Crocidura leucodon et Crocidura suaveolens sont signalées pour la première fois.

Le pourcentage de l'infection est relativement bas.

Avec des adiaspores peuvent être infectés des animaux de différents groupes systématiques. Sont observées des différences d'espèces par rapport à la fréquence et l'intensité de l'infection.

On a constaté des variations saisonnières, le plus haut degré d'infection étant noté pendant les mois de juin et de juillet. La dynamique saisonnière est très probablement conditionnée par les particularités climatiques et géographiques du biotope et par les biorythmes de l'hôte.

Il est à supposer que l'humidité du biotope joue un certain rôle sur la persistance et la propagation de l'infection.

Il semble que les conditions de vie des rongeurs sinantropes ne favorisent pas la contamination.

\section{Summary}

Adiasporomycosis in small wild mammals from Bulgaria.

After researches performed on 2057 specimens of wild mammals, originated from 3 regions of Bulgaria and belonging to 28 species, the A.A. found adiaspores in the 10 following species: Crocidura leucodon, C. suaveoles, Neomys anomalus, Sorex minutus, 
Apodemus agrarius, A. flavicollis, A. sylvaticus, Mus. musculus, Microtus arvalis, Clethrionomys glareolus. Among them, Crocidura leucodon and $C$. suaveolens are mentioned for the first time. Natural infection is relatively weak.

Experimentally, many variations are observed in frequency and intensity of infection between the differents systematical groups.

In natural conditions, the degree of infection depends on seasonal variations and specific biorythms of the host.

L'adiaspiromycose causée par Emmonsia crescens, Emmons et Jellison 1960, représente une mycose pulmonaire très répandue chez les petits mammifères. Dernièrement, elle est l'objet d'une recherche poussée dans plusieurs pays d'Europe, plus spécialement en Tchécoslovaquie, en France et en Union Soviétique (3, 4, 5, 6, 7, $10,12,13)$. Elle est établie chez un grand nombre de petits mammifères dans tous les pays où l'on a exécuté des recherches orientées: la Suède, la Norvège, la Finlande, l'Allemagne, la Suisse, la France, la Tchécoslovaquie, la Yougoslavie et l'Union Soviétique $(3,7)$.

En Bulgarie pour la première fois Roussakiev, Rositzki et al. informent de la présence d'adiaspores dans les poumons de quelques espèces de mammifères (11). Les recherches systématiques en la matière commencent en 1970, P. Pavlov et coll. (8, 9).

En 1970, 1971 et au début de 1972, nous avons exécuté des recherches mensuelles chez des petits mammifères provenant de différentes régions du pays. Nous nous sommes proposé d'examiner la propagation de l'infection, de suivre la dynamique saisonnière de l'infestation ainsi que d'élucider l'influence de certains facteurs écologiques sur l'évolution et sur l'incidence de l'infection.

\section{Matériel et méthodes.}

Nous avons examiné au total 2057 exemplaires de mammifères appartenant à 28 espèces : 8 espèces d'insectivores, 15 espèces de rongeurs et 5 espèces de rapaces (Tableau 1).

Les animaux examinés proviennent de 7 biotopes dans les environs de la réserve «Srebarna» (de la Bulgarie nord-est), 3 biotopes dans la montagne de Vitocha et les environs de la ville de Sofia et 1 biotope dans la montagne Sredna gora.

Les biotopes dans la région de la réserve «Srebarna s situés à 10-50 mètres d'altitude de la mer sont distribués de la façon suivante : 3 biotopes sont à proximité directe du lac. Ils sont couverts de végétation marécageuse et présentent une humidité élevée pendant toute l'année; 1 biotope est situé dans une forêt feuillue, à proximité du lac; 2 biotopes se trouvent dans des terres arables (champs et vignes) et sont caractérisés par une humidité considérablement plus basse ; 1 biotope est situé dans les locaux d'habitation et d'exploitation du village de Srebarna.

Les trois biotopes dans les environs de Sofia et de la montagne de Vitocha situés à 800-1 400 mètres au-dessus du niveau de la mer sont constitués, l'un par des terres 
TABLEAU I.

Liste des espèces d'animaux examinés pour la présence d'adiaspores d'Emmonsia crescens dans trois régions de Bulgarie

\begin{tabular}{|c|c|c|c|c|c|c|c|c|c|c|c|c|}
\hline Espèce & \multicolumn{3}{|c|}{ Srebarna } & \multicolumn{3}{|c|}{$\begin{array}{c}\text { Vitocha } \\
\text { exam. inf. \% }\end{array}$} & \multicolumn{3}{|c|}{$\begin{array}{l}\text { Sredna gora } \\
\text { exam. inf. \% }\end{array}$} & \multicolumn{3}{|c|}{$\begin{array}{r}\text { Total } \\
\text { exam. inf. }\end{array}$} \\
\hline $\begin{array}{l}\text { 1. Erinaceus rouma- } \\
\text { nicus } \ldots \ldots \ldots \ldots\end{array}$ & 3 & - & 一 & - & - & - & - & - & - & 3 & - & 一 \\
\hline 2. Talpa europaea .. & 1 & - & - & - & - & 一 & - & $-\cdot$ & - & 1 & - & 一 \\
\hline $\begin{array}{l}\text { 3. Crocidura leuco- } \\
\text { don ........... }\end{array}$ & 143 & 1 & 0,6 & 一 & - & 一 & - & - & - & 143 & 1 & 0,6 \\
\hline $\begin{array}{l}\text { 4. Crocidura suaveo- } \\
\text { lens ........... }\end{array}$ & 231 & 1 & - & 9 & - & - & 1 & 一 & - & 241 & 1 & 0,4 \\
\hline 5. Sorex araneus ... & 91 & - & - & 6 & - & 一 & 1 & - & - & 98 & - & 一 \\
\hline 6. Sorex minutus ... & 129 & 1 & 0,7 & 1 & - & 一 & - & - & - & 130 & 1 & 0,70 \\
\hline 7. Neomys anomalus & 170 & 4 & 2,3 & 6 & - & 一 & - & - & - & 176 & 4 & 2,20 \\
\hline 8. Neomys fodiens .. & - & - & - & 4 & - & 一 & - & - & - & 4 & - & 一 \\
\hline 9. Citellus citellus .. & 10 & - & - & - & - & 一 & - & - & - & 10 & - & 一 \\
\hline 10. Dyromys nitedula & 11 & - & - & - & - & 一 & - & - & - & 11 & - & 一 \\
\hline $\begin{array}{l}\text { 11. Micromys minu- } \\
\text { tus } \ldots \ldots \ldots \ldots \ldots\end{array}$ & 85 & - & - & - & - & 一 & - & - & - & 85 & - & 一 \\
\hline $\begin{array}{l}\text { 12. Apodemus sylvati- } \\
\text { cus ........... }\end{array}$ & 319 & 3 & - & 13 & - & 一 & 34 & 5 & - & 366 & 8 & 2,10 \\
\hline $\begin{array}{l}\text { 13. Apodemus flavicol- } \\
\text { lis } \ldots \ldots \ldots \ldots \ldots\end{array}$ & 145 & 3 & - & 2 & - & 一 & 7 & - & - & 154 & 3 & 1,90 \\
\hline 14. Apodemus agrarius & 252 & 4 & 1,5 & - & - & 一 & 一 & - & - & 252 & 4 & 1,5 \\
\hline 15. Mus musculus .. & 175 & 3 & 1,7 & - & - & - & 19 & - & - & 194 & 3 & 1,5 \\
\hline 16. Rattus norvegicus & 9 & - & - & 1 & - & 一 & 1 & - & - & 11 & - & 一 \\
\hline 17. Rattus rattus ... & 2 & - & - & 一 & 一 & 一 & 一 & - & 一 & 2 & - & 一 \\
\hline $\begin{array}{l}\text { 18. Mesocricetus aura- } \\
\text { tus ........... }\end{array}$ & 3 & - & - & - & - & 一 & - & - & - & 3 & - & 一 \\
\hline 19. Ondathra zibethica & 3 & - & - & - & 一 & 一 & - & - & - & 3 & - & 一 \\
\hline 20. Arvicola terrestris & 2 & - & - & - & - & - & - & - & - & 2 & - & 一 \\
\hline 21. Microtus arvalis. & 106 & 1 & 0,9 & - & - & 一 & - & - & - & 106 & 1 & 0,90 \\
\hline $\begin{array}{l}\text { 22. Clethrionomys gla- } \\
\text { reolus ........... }\end{array}$ & - & - & 一 & 4 & - & 一 & 2 & 1 & 一 & 6 & 1 & 一 \\
\hline $\begin{array}{l}\text { 23. Pitymys subterra- } \\
\text { neus } \ldots \ldots \ldots \ldots\end{array}$ & 31 & - & - & 3 & 一 & 一 & 2 & - & - & 36 & - & 一 \\
\hline 24. Meles meles .... & 1 & - & - & - & - & 一 & - & - & - & 1 & - & 一 \\
\hline 25. Mustela nivalis .. & 14 & - & - & 1 & - & - & - & - & - & 15 & - & 一 \\
\hline 26. Martes foina .... & 1 & - & - & - & - & 一 & - & - & - & 1 & - & 一 \\
\hline 27. Vulpes vulpes .... & 1 & 一 & - & - & - & 一 & - & - & - & 1 & - & 一 \\
\hline \multirow[t]{2}{*}{ 28. Putorius putorius } & 2 & - & - & - & - & - & - & - & - & 2 & - & - \\
\hline & 1940 & 17 & 3 & 50 & - & - & 67 & 6 & 8,9 & 2057 & 23 & 11 \\
\hline
\end{tabular}


arables et des prairies, l'autre par les rives d'un petit fleuve, dans la zone des villas autour de la ville et le troisième par une hétraie et les moraines du fleuve de Boyana.

Le biotope de Sredna gora est situé dans une forêt mixte, constituée d'espèces feuillues et conifères à 1100 mètres d'altitude.

Nous avons disséqué les animaux sur le terrain en prélevant à partir des poumons des échantillons pour effectuer des ensemencements sur les milieux de culture de Sabouraud et CC-agar-Difco. Une partie du poumon a été observée directement pour détecter la présence d'adiaspores, avec compresseur pour la trichineloscopie, le reste étant fixé dans l'alcool à $70^{\circ}$ et examiné par la méthode de Doby et BoisseauLebreuil (2).

De plus, pour isoler l'agent du sol pendant les mois d'hiver 1971-1972 nous avons examiné 105 échantillons de sol prélevés dans les trous des petits mammifères dans la région de la réserve «Srebarna».

\section{Discussion.}

Comme il est montré dans le tableau 1, de toutes les 28 espèces de mammifères sauvages examinées, les exemplaires infectés par des adiaspores d'Emmonsia crescens appartenaient à 10 espèces : Cricidura leucodon $0,6 \%$, Crocidura suaveolens $0,4 \%$, Neomys anomalus 2,2 \%, Sorex minutus 0,7\%, Apodemus agrarius 1,5\%, Apodemus flavicollis 1,9 \%, Apodemus sylvaticus 2,1 \%, Mus musculus 1,5\%, Microtus arvalis $0,9 \%$ et Clethrionomys glareolus 1 de 6 examinés. Parmi eux, Microtus arvalis et Sorex minutus sont annoncés pour la première fois comme des hôtes d'Emmonsia crescens en Bulgarie. Crocidura leucodon et Crocidura suaveolens ne sont pas rapportées jusqu'à maintenant comme des porteurs d'adiaspores dans la littérature mondiale.

Les recherches réalisées et les résultats obtenus nous donnent lieu de faire les constatations suivantes quant à la réceptivité naturelle des mammifères sauvages répandus dans les régions examinées, la dynamique saisonnière de la fréquence d'infestation, ainsi qu'en ce qui concerne l'influence de quelques facteurs écologiques, biologiques et géographiques.

Tout d'abord, nous voudrions attirer l'attention sur le bas pourcentage d'infection par rapport aux données des auteurs tchèques $(6,10)$, français $(3,4)$ et soviétiques $(12,13)$. Nos données sont plus voisines des résultats obtenus par Doby et coll. pour la péninsule ibérique (5). Il est possible que cela soit en liaison avec les particularités climatiques et géographiques du pays.

En ce qui concerne la réceptivité naturelle des différentes espèces d'hôtes, les résultats de nos recherches ne font pas voir qu'il existe des différences essentielles à cet égard. Les données du tableau 2 montrent qu'avec les adiaspores d'Emmonsia crescens peuvent être infectés des animaux de différents groupes systématiques même au cas où les conditions écologiques varient d'un groupe à l'autre. D'autre part, la fréquence de l'infection et l'intensité montrent des différences déterminées du point de vue de l'espèce. Parmi les mammifères insectivores, ce sont Noomys anomalus qui sont 


\section{Tableau II.}

Liste des espèces d'animaux infectés avec des adiaspores d'Emmonsia crescens et $\%$ de l'infection en Bulgarie

\begin{tabular}{|c|c|c|c|c|c|c|}
\hline \multirow{2}{*}{ Espèce hôte } & \multicolumn{3}{|c|}{$\begin{array}{l}\text { Selon des données de P. Pavlov } \\
\text { et coll., } 1970\end{array}$} & \multicolumn{3}{|c|}{ Nos recherches } \\
\hline & examinés & infectés & $\%$ & examinés & infectés & $\%$ \\
\hline $\begin{array}{l}\text { 1. Crocidura leuco- } \\
\text { don } \ldots \ldots \ldots \ldots\end{array}$ & - & 一 & - & 143 & 1 & 0,63 \\
\hline $\begin{array}{l}\text { 2. Crocidura suaveo- } \\
\text { lens } \ldots \ldots \ldots \ldots .\end{array}$ & - & 一 & - & 241 & 1 & 0,40 \\
\hline 3. Neomys anomalus & 27 & 1 & 3,70 & 176 & 4 & 2,20 \\
\hline 4. Neomys fodiens .. & 5 & 3 & - & - & - & - \\
\hline 5. Sorex minutus .. & - & - & - & 129 & 1 & 0,70 \\
\hline 6. Apodemus agrarius & 24 & 2 & 8,00 & 252 & 4 & 1,50 \\
\hline $\begin{array}{l}\text { 7. Apodemus flavicol- } \\
\text { lis .............. }\end{array}$ & 34 & 8 & 23,50 & 154 & 3 & 2,10 \\
\hline $\begin{array}{l}\text { 8. Apodemus sylvati- } \\
\text { cus } \ldots \ldots \ldots \ldots\end{array}$ & 125 & 8 & 6,40 & 366 & 8 & 1,90 \\
\hline 9. Mus musculus ... & 15 & 3 & - & 194 & 3 & 1,50 \\
\hline 10. Rattus rattus ... & 2 & 1 & - & - & - & 一 \\
\hline $\begin{array}{l}\text { 11. Clethrionomys gla- } \\
\text { reolus .......... }\end{array}$ & 8 & 5 & - & 6 & 1 & 一 \\
\hline 12. Microtus arvalis .. & - & 一 & - & 106 & 1 & 0,90 \\
\hline $\begin{array}{l}\text { 13. Pitymys subterra- } \\
\text { neus } \ldots \ldots \ldots \ldots\end{array}$ & 5 & 1 & - & - & - & - \\
\hline 14. Spalax leucodon .. & 38 & 2 & 5,30 & - & - & - \\
\hline
\end{tabular}

Note: Les données de P. Pavlov et coll., 1970 concernent les recherches réalisées pendant les mois de mars, de mai et de juin 1970.

Nos recherches ont été exécutées pendant toute l'année.

plus souvent et plus intensivement infectés d'adiaspores par rapports aux espèces du genre Crocidura et Sorex minutus. Chez les rongeurs, la même tendance est observée en comparant l'infection des trois espèces du genre Apodemus avec les autres représentants de Murinae et Microtinae.

Les données littéraires sur le problème des variations saisonnières dans la fréquence d'infection sont contradictoires. Tandis que pour la Tchécoslovaquie, Dvorak et coll. (1967) trouvent des animaux infectés d'adiaspores seulement pendant les mois de mars, d'avril et de mai, Prokopic (1971) observe le plus haut pourcentage d'infection pendant les mois d'avril et de septembre et le plus bas pourcentage 
pendant les mois d'hiver et d'été. D'autre part, Doby et coll. (1971) ne trouvent pas de variations saisonnières pareilles dans les conditions françaises.

Les recherches mensuelles effectuées chez nous dans la région de la réserve «Srebarna» (de la Bulgarie nord-est) à partir du mois de juin 1970 jusqu'en février 1972 font voir que le taux et l'intensivité d'infection est le plus élevé pendant les mois de juin et de juillet. Mais il convient cependant de noter que nous avons trouvé aussi des animaux infectés pendant les autres mois de l'année.

Nos observations réalisées dans les différentes parties du pays nous autorisent à conclure qu'il existe certaines différences dans la dynamique saisonnière d'infection 'conditionnées par les particularités climatiques et géographiques de la région considérée. En même temps, il importe de garder à l'esprit les particularités dans la biologie de chaque espèce hôte.

Les recherches comparatives dans les biotopes différents montrent certaines différences selon l'habitat de l'hôte. Nous avons observé des animaux infectés dans tous les biotopes. Soulignons cependant que la fréquence de l'infestation est plus élevée chez les biotopes présentant une humidité considérable.

En liaison avec ce que nous venons d'exposer, il est intéressant de noter que les rongeurs sinantropes des bâtiments d'habitation ou d'exploitation du village de Srebarna ne sont pas infectés. Ainsi par exemple, des 46 exemplaires de Mus musculus, provenant de ce biotope, pas un seul n'a pas été contaminé. En même temps, dans les populations de Mus musculus constituées dans les terres arables loin de la localité, nous avons trouvé 3 animaux infectés sur les 129 examinés (2,3\%). Il semble que les conditions de la vie dans les habitations ne favorisent pas le développement de l'infection. A des pareilles conclusions arrivent aussi Doby et coll. (1970).

Nos recherches montrent qu'il n'existe pas de dépendance entre l'infection avec les adiaspores d'Emmonsia crescens et l'altitude, ce qui est en accord avec les résultats établis par d'autres chercheurs $(1,3,8,9)$.

Notons en guise de conclusion que nos expériences visant à isoler l'agent du sol à partir des trous des petits mammifères n'ont pas donné des résultats positifs.

\section{Bibliographie}

1. Blažsek (K. M.) and Daniel (1971). - Adiaspiromycosis caused by Emmonsia crescens in small mammals from Hindu kush Montains (W. Pakiston). Folia parasitologica (Praha), 17, 34.

2. Doby (J.-M.) et Boisseau-Lebreuil (M.-T.), 1969. - Méthode de recherche par digestion et homogénéisation des spores d'Emmonsia crescens, agent de l'adiaspiromycose dans le poumon de petits mammifères. Ann. parasit. hum et comp., 44, 785-788.

3. - - - et Beaucournu (J.-C.), 1970. - Nouveaux hôtes pour Emmonsia crescens, Emmons et Jellison, agent de l'adiaspiromycose. Bull. soc. Path. exot., 63, 324-334.

4. —, - et Rault (B.), 1971. - L'adiaspiromycose par Emmonsia crescens chez les petits mammifères sauvages en France. Mycopath. Mycol. appl., 44, 2, 107-115. 
5. - - - et Piédade Guerreiro (J.), 1971. - Adiaspiromycose chez les petits mammifères sauvages dans la péninsule ibérique. Anais da escola Nacional de saude publica e de Medicina tropical, 5, 55-57 (Lisboa).

6. Dvořak (J.), OtčEnAšEk (M.) and Procopitch (J.), 1967. - Seasonal incidence of adiaspores of Emmonsia crescens, Emmons and Jellison, 1960 in wild living animals. Mycopath. mycol. appl., 34, 71.

7. - 1969. - Survey of animals examined on the presence of adiaspores of Emmonsia crescens, Emmons and Jellison, 1960. Folia parasitologica (Praha), 16, 349-355.

8. Pavlov (P.), Guenov (T.) and Prokopič (J.), 1971. - Studies on the incidence of adiaspiromycosis in Bulgaria. Veterinary science, 8, 9-13.

9. —, - et —, 1971. — Recherche sur la propagation de l'adiaspiromycose en Bulgarie. Bull. Soc. Path. Exot., 64, 458-464.

10. Prokopič (J.), 1971. - The Distribution of adiaspiromycosis in small mammals of the Czech Socialis Republic. Folia parasitologica (Praha), 18, 323.

11. Roussakiev (M.), Rossitzki (B.), Dinev (T.), Angelova (V.), Heiny (S.), Christova (T.), Kolman (J.), Mateev (D.), Minarge (J.), Pissarska (P.), Serbova (S.), Shebek (Z.), Zlatanov (Z.), 1968. - Investigation on natural focci of infections in Batova river, Bulgaria. National congress of Parasitology, Sofia, 122-124.

12. Sharapov (V. M.), 1969. - Adiaspiromycosis in USSR. Izvestia sibir. Otdel. Acad. SSSR, 86-95.

13. - 1970. - Adiaspiromycosis in mustelidae. Transaction of the IX international congress of game biologist, Moscow, 662-665. 